\title{
Ultrasound Tissue Characteristics of Diabetic Muscles and Tendons: Associations with Strength and Laboratory Blood Tests
}

\author{
C.Y. Kuo ${ }^{1}$, W. N. Lee ${ }^{2}$, S. S. M. Fong ${ }^{3}$, P. Chaiyawat ${ }^{4}$, G. Bashford ${ }^{5}$, Tiffany T.F. Shih ${ }^{6,7}$, L. \\ M. Chuang ${ }^{8,9}$, H. K. Wang ${ }^{1,10}$ \\ 1 School and Graduate Institute of Physical Therapy, College of Medicine, National Taiwan University, Taipei, \\ Taiwan \\ 2 Department of Electrical and Electronic Engineering and Biomedical Engineering Programme, The University of \\ Hong Kong, Hong Kong, China \\ 3 School of Public Health, The University of Hong Kong, Pokfulam, Hong Kong, China \\ ${ }^{4}$ Faculty of Physical Therapy, Mahidol University, Nakhon Pathom, Thailand \\ ${ }^{5}$ Department of Biological Systems Engineering, University of Nebraska-Lincoln, Lincoln, Nebraska, USA \\ 6 Department of Radiology, College of Medicine, National Taiwan University, Taipei, Taiwan \\ 7 Department of Medical Imaging, National Taiwan University Hospital, National Taiwan University, Taipei, Taiwan. \\ 8 College of Medicine, National Taiwan University, Taipei, Taiwan \\ 9 Division of Endocrinology and Metabolism, Department of Internal Medicine, National Taiwan University \\ Hospital, Taipei, Taiwan \\ ${ }^{10}$ Department of Physical Medicine and Rehabilitation, National Taiwan University Hospital, National Taiwan \\ University, Taipei, Taiwan
}

\section{CORRESPONDING AUTHOR:}

\section{Hsing-Kuo Wang}

Medicine, National Taiwan University

Sports Physiotherapy Laboratory

School and Graduate Institute

of Physical Therapy

College of Medicine

Xuzhou Road, Zhongzheng District

Taipei, Taiwan, 100

E-mail: hkwang@ntu.edu.tw

DOI:

10.32098/mltj.03.2020.07

LEVEL OF EVIDENCE: 3B

\section{SUMMARY}

Background. This study sought to compare the ultrasound tissue characteristics of the muscles (including muscle thickness, echo intensity, and stiffness) and tendons (including thickness, stiffness, and peak spatial frequency radius (PSFR)) of participants with or without diabetes mellitus. Moreover, the study sought to determine any relationships between the muscle stiffness and strength or tendon PSFR and the results of blood tests, including the glycation or lipid profiles, of the diabetics.

Methods. Twenty-three participants with type 2 diabetes mellitus and nineteen controls without a history of diabetes were recruited.

Results. The diabetic muscles exhibited less thickness $(P=0.024)$, greater echo intensity $(P=0.033$ and 0.002$)$, and lower muscle stiffness $(P=0.015$ and 0.009$)$ than the control muscles. Furthermore, the diabetic tendons exhibited a lower PSFR ( $P$ ranged between 0.037 and $<0.001$ ). There were correlations between the resting stiffness of the gastrocnemius muscle and the height of heel lifting $(r=0.450, P=0.031)$, between the PSFR in the patellar tendon and the hemoglobin A1c level $(r=-0.539, P=0.017)$, and between the PSFR in the Achilles tendon and the high-density lipoprotein cholesterol level $(r=0.545$, $P=0.019$ ).

Conclusions. The diabetic muscles and tendons exhibited morphomechanical changes associated with force capacity or markers of insulin resistance. Clinical applications of musculoskeletal ultrasound techniques to diabetics include using them to design exercise strategies and for microstructural screening.

\section{KEY WORDS}

Diabetes; musculoskeletal ultrasound; tissue characteristics; muscle; tendon. 


\section{BACKGROUND}

Diabetes mellitus (DM) is a major cause of mortality and functional disability worldwide, and the complications resulting from it include microvascular diseases and musculoskeletal disorders (1). Long-term hyperglycemia and impaired lipid metabolism are considered to be among the causes of the diabetic myotendinous disorders, which may be accompanied by morphological (structural) changes, dysfunction, and pain (2). In a magnetic resonance imaging study focusing on morphology, diabetic calf muscles were found to exhibit atrophy with fat infiltration, loss of muscle mass, increased fatigability, and a greater reliance on glycolytic metabolism in comparison to the muscles of controls without diabetes (3). In a study observing the micro-structure and morphology of the Achilles tendons of patients with long-term DM using an electron microscope, it was reported that such tendons have reduced fiber diameter, increased collagen fiber bulk density, and abnormal fiber morphology (4). These findings themselves suggest possible mechanisms underlying the findings of other reports indicating that (a) the hyperechoic appearance of diabetic muscles in ultrasound imaging correctly predicted diabetes in 70 of 79 patients (5); (b) long-term diabetes is associated with a high prevalence of chronic Achilles tendinopathy, including hypoechogenicity, entheseal thickening, and enthesophytes (2,6); and (c) diabetic tendons demonstrate inferior elasticity (Young modulus), maximum load, and stiffness levels $(7,8)$. Theoretically, these respective muscle morphological and tendon microstructural changes are the causes of diabetic muscle force compromise, in addition to being evidence of diabetic complications in tissues with low reparative capacity. However, the studies referenced above left unanswered questions about (a) whether atrophy with fat infiltration in diabetic muscles leads to a reduction of muscle stiffness, which is an elemental determinant of force development; (b) whether abnormal morphology and disorganization of the collagen fibrils in the diabetic tendon could reflect on the primary fiber bundle, the basic unit of transmitting and resisting tensile stresses in the tendon (9); and (c) whether the aforementioned morphomechanical changes are associated with force production within the myotendinous complex or the profiles of blood biomarkers representing the pathology of diabetes. Further studies are thus required to answer the above questions and establish clinical protocols for periodic evaluations to monitor diabetes-related myotendinous deteriorations.

High-resolution B mode ultrasonography and elastography have been determined to be reliable techniques for measuring the echo intensity (EI), mechanical stiffness (elastic modulus), and morphologies of skeletal muscle. Skeletal muscle stiffness is determined using shear-wave elastogra- phy according to the tension produced by muscle contraction and mechanical properties along the muscle fibers (10). In addition, changes in the physical characteristics of the microstructure of the tissue can be quantified by detecting the energy behavior of scattering of the B-mode image structure with spectral analyses of the waveform data of ultrasonic radiofrequency signals, such as a high proportion of energy at low frequencies as the scatter size increases (11). Other studies have shown the microstructural changes that occur in tendinopathy via the low values of peak spatial frequency radius (PSFR), which consists of the distance from the origin to the spatial frequency peak of greatest amplitude on the 2-D fast Fourier transform spectrum, exhibited in symptomatic subjects (12). It was inferred that, the ultrasound wavelength ranged between $0.32 \mathrm{~mm}(320 \mu \mathrm{m})$ and $0.13 \mathrm{~mm}(130 \mu \mathrm{m})$, with a $5-12 \mathrm{MHz}$ probe being able to detect the scattering from the structures forming a primary bundle or subfascicle (15-400 $\mu \mathrm{m}$ in diameter), which was mainly composed of collagen fibrils (with diameters of 30-300 nm) and small collagen fibers (with diameters of $1-300 \mu \mathrm{m})(9)$. Collectively, DM-associated deteriorations in muscle mechanical properties and tendon microstructures respectively represent the compromised capacity of muscle force production and the pathologies of diabetes and can be assessed by elastographic and ultrasonic tissue characterization using spectrum analysis. However, profiles of the above characteristics have not been reported or completely analyzed in past studies.

The aim of the present study, therefore, was two-fold. First, to measure and compare the ultrasound tissue characteristics of the muscles (including muscle thickness, EI, and stiffness) and tendons (including thickness, stiffness, and PSFR) of participants with DM who demonstrated negative results in peripheral vascular and nerve screening testing to those of participants without DM. In addition, the study sought to determine any relationships between the muscle stiffness and strength or tendon PSFR and the results of blood tests, including the glycation or lipid profiles, in the participants with DM. We hypothesized that there would be differences in the aforementioned ultrasonic tissue characteristics of the myotendinous complex of the lower extremities in the DM patients when compared to controls. Furthermore, it was hypothesized that, in the DM group, the stiffness in certain muscles (namely, the vastus lateralis and medial gastrocnemius) and the PSFR in the tendons (namely, the patellar and Achilles tendons) would be associated, respectively, with muscle strength results and blood test results, including hemoglobin $\mathrm{A} 1 \mathrm{c}(\mathrm{HbA1c})$ and high-density lipoprotein cholesterol (HDL-C) levels. The HbA1c and HDL-C levels were chosen because abnormalities in serum lipid profiles and $\mathrm{HbA} 1 \mathrm{c}$ levels represent markers of insulin resistance 
and the risk for micro- and macroangiopathies in known type 2 diabetic patients (13).

\section{MATERIALS AND METHODS}

\section{Subjects and study design}

This study utilized a cross-sectional design that compared a group of diabetic participants with another group of participants who were matched in terms of physical characteristics but did not have a history of DM. This study was approved by the institutional review board of National Taiwan University Hospital (reference no. 201703035RINC) and meets the ethical standards of the journal (14). All of the eligible diabetic and control participants were recruited from an outpatient clinic of a university-affiliated hospital. Written informed consent was obtained from all the participants prior to participation, and details that might disclose the identity of the participants have been omitted. All the diabetic participants were between 30 and 80 years old and were recruited based on the 2018 criteria of the American Diabetes Association (15) and their negative results on peripheral vascular or nerve screening tests, including their ankle-brachial index results $(<0.9$ or $>1.3)$, Semmes Weinstein monofilament examination results (any one of 4 sites was insensate on each foot), and $128-\mathrm{Hz}$ tuning fork vibration test results (perceptions - 5 seconds). The participants in the control group who were matched in terms of physical characteristics to the DM group were recruited from the hospital department of volunteer and charity workers. The inclusion criteria for the control participants included no history of DM. Any of the potential participants were excluded if they had risk factors for structural weakness in the myotendinous complex, including a chronic kidney injury treated with hemodialysis, hyperuricemia, rheumatoid arthritis, systemic lupus erythematosus, osteogenesis imperfecta, musculoskeletal tuberculosis, parathyroidism, hypothyroidism, or alkaptonuria.

\section{Experimental protocol}

The participants were asked to change into a hospital gown if their clothes prevented ultrasonographic measurements of the rectus femoris, vastus lateralis, quadriceps tendon, patellar tendon, medial gastrocnemius, or Achilles tendon. They were instructed to rest for 10 minutes and fill out a brief questionnaire including questions regarding their DM history. Ultrasound image acquisition was conducted in the medical imaging department of the university hospital with an Aixplorer ${ }^{\circledR}$ system (Supersonic Imaging, Aix-en-Provence, France) coupled with an SL15-4 probe.
The Young's modulus, grayscale image features, thickness, and PSFR of the aforementioned muscles or tendons were measured in a longitudinal view. The elasticity measurements using a round region of interest (ROI) with an approximately 4-7 $\mathrm{mm}$ diameter were recorded with the mean resting Young's modulus within the ROI on the same aforementioned muscles and tendons. During each elasticity measurement, the ultrasound transducer was kept stationary for $10 \mathrm{~s}$ during the acquisition of the image (figure $\mathbf{1}$ A-F). To standardize and optimize the image quality and to avoid variability in the B-mode images, the gain was adjusted to a default set of control settings $(46-48 \%)$, and the focus area was increased to maximum and kept consistent across all the participants to adjust for differences in muscle size among the participants. Muscle thickness assessed by $\mathrm{B}$-mode ultrasound was measured as the distance between the superficial and deep aponeuroses at the mid-line of the image, while the tendon thickness was determined by the maximum anteroposterior diameter of the tendons. The measurements for the rectus femoris and vastus lateralis muscles (on the lower third of the thigh), quadriceps tendon, and patellar tendon were conducted with the participant positioned in a supine position with the knee flexed at $60^{\circ}$, with this position maintained by pillows placed under the tested knee. Ultrasound measurements of the quadriceps and patellar tendons were respectively conducted at $1.5 \mathrm{~cm}$ above the superior border of the patella and in the area around the middle portion of the patellar tendon. For measurements of the proximal third of the gastrocnemius medialis muscle (i.e., the region between the lateral malleolus of the ankle and the lateral condyle of the knee) and the mid-portion of the Achilles tendon (i.e., the region $2-4 \mathrm{~cm}$ proximal to the calcaneal insertion), each subject lay prone (face down) on an examination bed with both ankles hanging over the edge of the bed, with the foot being placed at an angle of $90^{\circ}$ to the tibia.

B-mode ultrasound images were stored as jpeg files and transferred to a computer for processing regarding the muscle EI and tendon PSFR. The mean pixel intensity (i.e., the EI) of the selected muscle regions was obtained by gray-scale analysis using the standard histogram function in Image-J (ImageJ, U. S. National Institutes of Health, Bethesda, Maryland, USA) without any bone, aponeurosis, or surrounding fascia. The mean EI values for the different regions of muscle were expressed in values ranging between 0 and 256 (0: black; 256: white). The measurements of tendon PSFR were conducted using a protocol similar to that used by Kulig et al. (12), including the use of custom image analysis programs written in MATLAB (Mathworks, Natick, MA, USA). A quadrilateral-shaped ROI enclosing a maximum tendon area corresponding to the mid-substance 

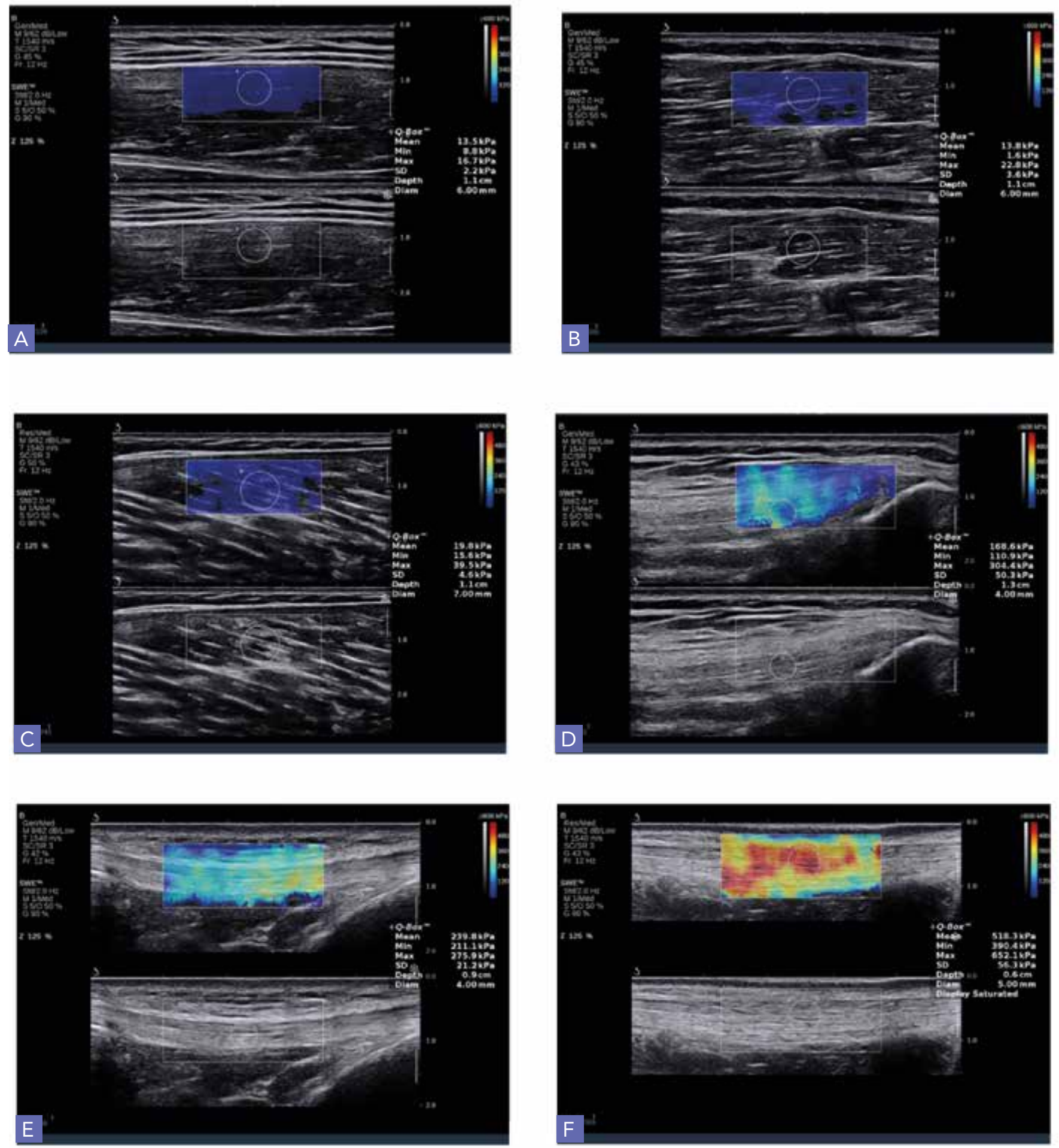

Figure 1. The measurements of and Young's modulus (shear-wave velocity) of the rectus femoris muscle (A), vastus lateralis muscle (B), medial gastrocnemius muscle (C), quadriceps tendon (D), patellar tendon (E), and Achilles tendon (F). 
of the tendon without the distal and proximal image (i.e., the distal and proximal tendon curvature) was selected for each PSFR measurement (12) (figure 2 A-C). Within the ROI, the PSFR in every $2 \mathrm{~mm}$-square kernel ( $32 \times 32$ pixel) circumscribed by the ROI was analyzed and averaged for each image (12).

After the ultrasonographic measurements, the participants were assessed in two strength testing sessions, the first of which involved the knee extension exercise and the second of which involved the heel raising exercise. There was a five-minutes interval of rest between the two sessions. During the testing sessions, each participant was first instructed to perform the maximum isometric knee extension in a sitting position and then the one-legged heel raising in a standing position using his or her bodyweight. The knee testing was completed with a customized wheelchair with an affixed load cell attached to the ankle cuff just above the medial malleolus, with the lumbar-hip and knee joints respectively positioned at $100-110^{\circ}$ and $60^{\circ}$ extensions (with $0^{\circ}$ corresponding to the complete extension). Both upper limbs of each participant were kept crossed in front of the participant's chest. In the heel raising testing, each participant was asked to stand on the tested leg and instructed to perform maximal heel lifting. The participant was allowed to touch a wall with an index finger at the shoulder level to maintain a steady one-leg standing position. The maximal force for the knee extension exercise and height for the heel raising exercise were recorded.

\section{Laboratory tests}

Results of blood tests (including tests of HbA1c, triacylglycerol (TG), total cholesterol, low-density cholesterol (LDL), and HDL-C levels) were collected over a period of approximately 6 months. The HbA1c and HDL-C levels were enrolled for the correlation analyses.

\section{STATISTICAL ANALYSIS}

The Shapiro-Wilk test was used to determine if the data for all the variables were normally distributed. The Mann-Whitney $U$ test was used to analyze the differences between the diabetic tendons and non-diabetic tendons. Spearman's rank correlation coefficients were calculated to determine whether, respectively, the muscle Young's modulus and force production capacities (i.e., the knee extension torque and the heel lifting height) and the tendon microstructural characteristics and laboratory blood test results (HbA1c and HDL-C levels) were related. All of the analyses were performed using SPSS 22.0 for Windows (SPSS Inc, Chicago, IL, USA), with the $\alpha$ level set at 0.05 .
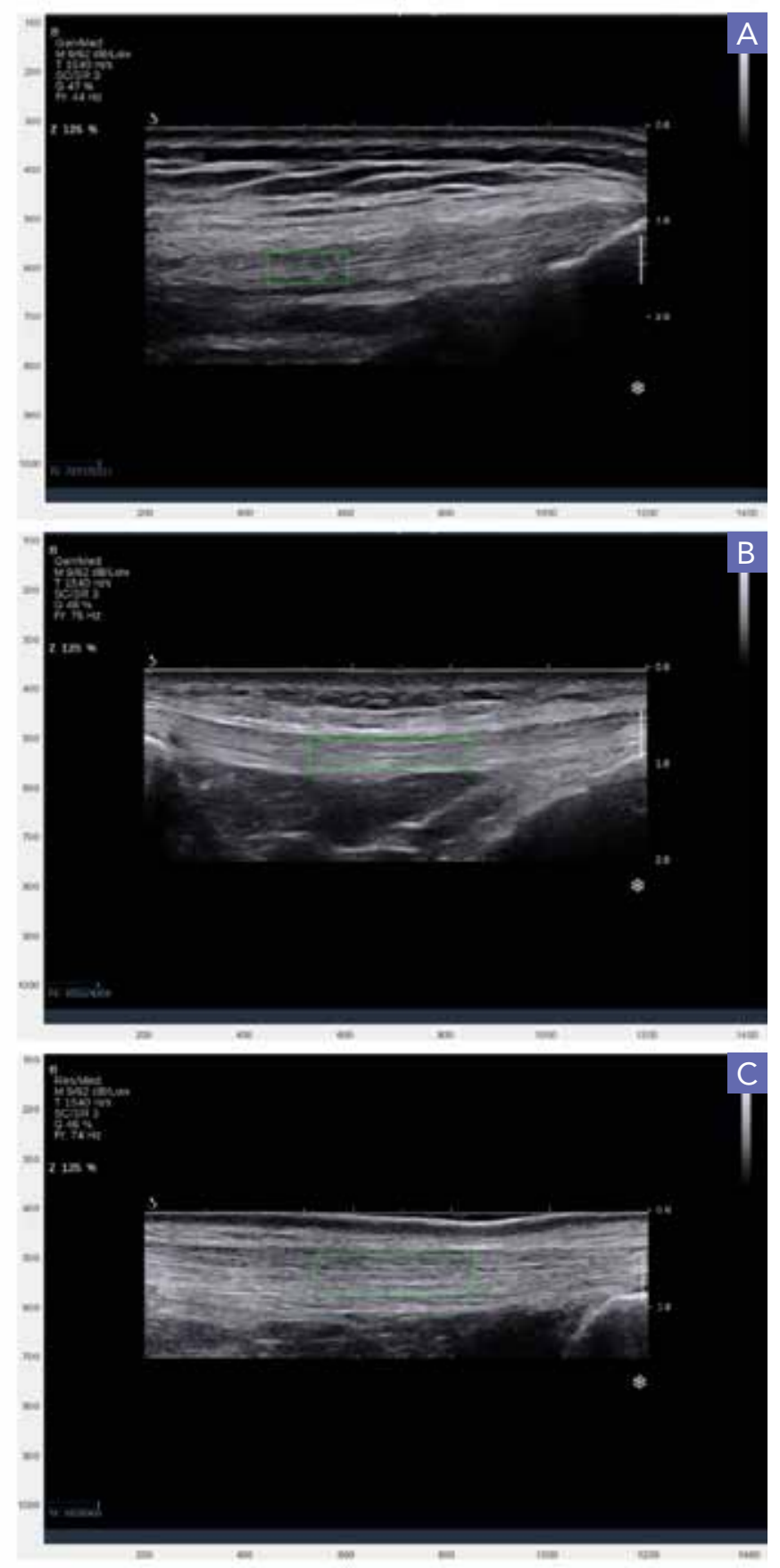

Figure 2. The measurements of peak spatial frequency radius (PSFR) for the quadriceps (A), patellar (B), and Achilles (C) tendons. 


\section{RESULTS}

\section{Participants}

Twenty-three participants with type $2 \mathrm{DM}$ and nineteen physically matched controls without a history of diabetes were recruited. The characteristics of the participants are summarized in Table 1. None of the diabetic participants had shown complications or signs of high risks of peripheral artery disease or neuropathy, such as the loss of the sensation of touch (table I). There were no significant differences in age or gender between the diabetic and control groups (both $P>0.05$ ), while the height of heel lifting was found to be greater in the controls $(P<0.001)$. The blood laboratory test results for the diabetic participants ranged between normal and moderate hypertriglyceridemia (TGs between 150-499 mg/dL), between normal and acceptable LDL levels (100-129 mg/dl), and between low (HDL $<50 \mathrm{mg} / \mathrm{dl}$ ) and normal HDL levels (16). The investigated diabetic muscles exhibited less thickness $(P=0.024)$, greater EI $(P=0.033$ and 0.002$)$, and lower muscle stiffness $(P=0.015$ and 0.009$)$ when compared to the control muscles. In addition, the diabetic tendons exhibited a lower PSFR ( $P$ ranged between 0.037 and $<0.001$ ) (table II); however, this was not consistent with the thickness and material stiffness results observed (all P>0.05) (table
III). There were correlations between the resting stiffness of the gastrocnemius muscle and the height of heel lifting $(r=0.490, P=0.021)$, between the PSFR in the patellar tendon and the HbA1c level $(r=-0.539, P=0.017)$, and between the PSFR in the Achilles tendon and the HDL-C level $(\mathrm{r}=0.545, P=0.012)$.

\section{DISCUSSION}

The results of this study verified our hypotheses and demonstrated that diabetes has effects on the morphomechanical properties of the muscles and tendons, as well as their associations with, respectively, plantarflexor muscle strength and blood sugar/lipid levels. These findings expand the existing knowledge of how long-term hyperglycemia and impaired sugar/lipid metabolism impact the mechanical properties and micro-structure, respectively, in muscles and tendons. They also provide further information regarding possible mechanisms of diabetic muscle weakness and tendinopathy genesis in type $2 \mathrm{DM}$ patients. Moreover, they highlight exercise strategies for early detected or well-controlled diabetes patients without significant signs of peripheral vascular disease and neuropathy aimed at preventing functional disability and tendon pain. In addition, they also indicate the potential contributions of ultrasound techniques in

Table I. Physical characteristics, laboratory test results, and muscle strength levels of the participants with and without diabetes mellitus.

\begin{tabular}{|c|c|c|c|}
\hline & Diabetic group $(\mathrm{N}=23)$ & $\begin{array}{c}\text { Non-diabetic group } \\
(\mathrm{N}=19)\end{array}$ & P value \\
\hline Age (in years) & $65(51-70)$ & $65(45-70)$ & 0.551 \\
\hline History (in years) & $9.5(3.0-23.0)$ & NA & \\
\hline $\mathrm{HAb} 1 \mathrm{C}(\%)$ & $7.3(5.9-10.5)$ & NA & \\
\hline LDL-C (mg/dL) & $94.0(51.0-132.0)$ & NA & \\
\hline $\mathrm{HDL}-\mathrm{C}(\mathrm{mg} / \mathrm{dL})$ & $49.5(33.0-66.0)$ & NA & \\
\hline Ankle-brachial index - right side & $1.2(1.1-1.3)$ & NA & \\
\hline Ankle-brachial index - left side & $1.2(1.1-1.3)$ & NA & \\
\hline Vibration test - left foot & $7.0(6.0-8.0)$ & NA & \\
\hline Knee isometric extension $(\mathrm{kg})$ & $21.0(8.96-45.7)$ & $22.6(16.2-34.2)$ & 0.336 \\
\hline Heel raising height $(\mathrm{cm})$ & $10.0(6.0-12.5)$ & $11.8(7.2-14.1)$ & $<0.001$ \\
\hline
\end{tabular}

Abbreviations: NA, not available. Results are presented as median values, with the range between the minimum and maximum values in the parentheses. 
Table II. Ultrasonic tissue characteristics of muscles in the participants with and without diabetes mellitus.

\begin{tabular}{|c|c|c|c|}
\hline & $\begin{array}{l}\text { Diabetic group } \\
\quad(\mathrm{N}=23)\end{array}$ & $\begin{array}{l}\text { Non-diabetic group } \\
\qquad(\mathrm{N}=19)\end{array}$ & $P$ value \\
\hline \multicolumn{4}{|l|}{ Muscle thickness (cm) } \\
\hline Vastus lateralis & $1.52(1.15-1.98)$ & $1.48(0.89-2.31)$ & 0.781 \\
\hline Medial gastrocnemius & $1.31(0.95-1.91)$ & $1.59(0.93-2.23)$ & $0.024 *$ \\
\hline Vastus lateralis & $84.65(50.80-122.00)$ & $66.6(38.65-116.63)$ & 0.067 \\
\hline Medial gastrocnemius & $80.14(34.29-113.44)$ & $50.69(28.35-109.53)$ & $0.002 *$ \\
\hline \multicolumn{4}{|c|}{ Mechanical stiffness (kilopascal) } \\
\hline Rectus femoris & $12.10(8.20-65.60)$ & $14.90(9.70-68.00)$ & 0.197 \\
\hline
\end{tabular}

Results are presented as median values, with the range between the minimum and maximum values in the parentheses. * means a significant difference. A. U. means arbitrary unit.

Table III. Ultrasonic tissue characteristics of tendons in the participants with and without diabetes mellitus.

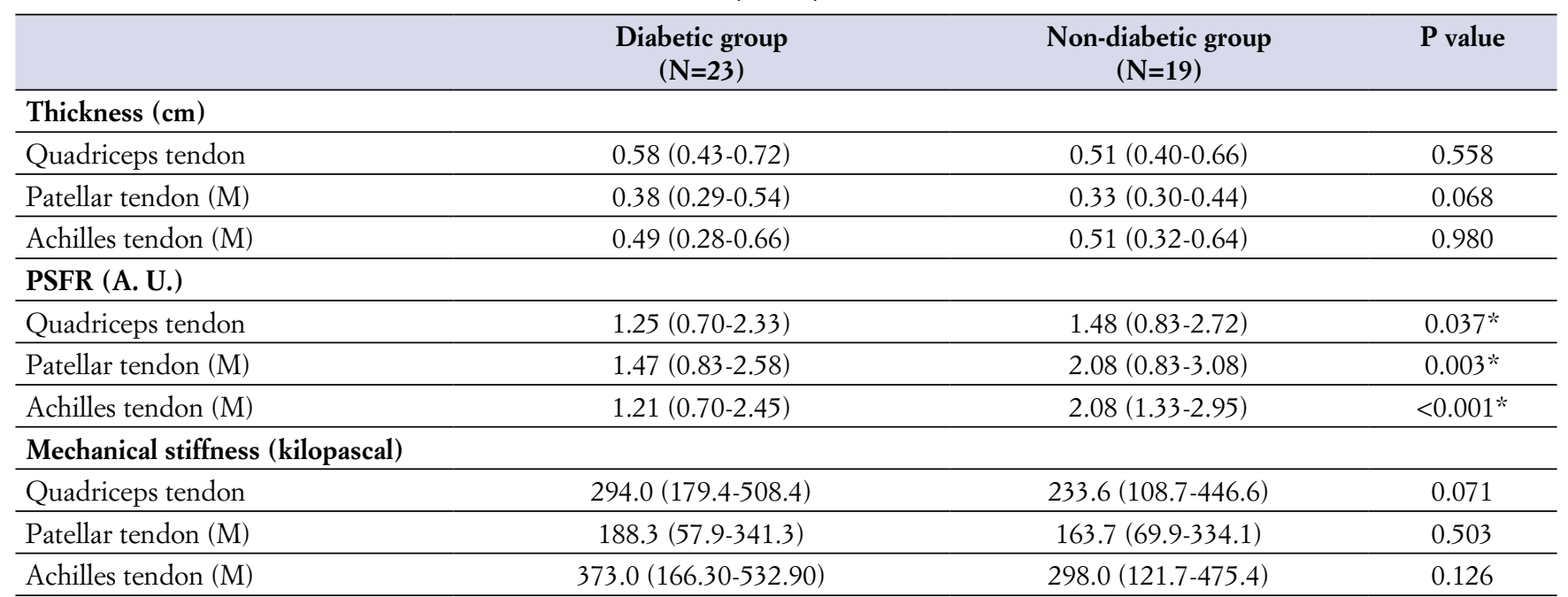

Results are presented as median values, with the range between the minimum and maximum values in the parentheses. * means a significant difference. $\mathrm{M}$ means the middle portion of the tendon. A. U. means arbitrary unit.

assessing muscle tissue characteristics and screening tendon pathogenesis in patients with metabolic disorders.

In the present study, the ultrasonic tissue characteristic results for diabetic muscles at rest indicated that in type 2 DM patients without peripheral vascular and neuropathic signs, the main deterioration of skeletal muscles in the lower extremities included decreases of muscle mass (reduced thickness), the accumulation of intramuscular fat (increased EI), and the reduction of the mechanical proper- ty (Young's modulus or shear-wave velocity). These results are consistent with previous studies that reported that (a) leg muscle thickness levels are reduced in diabetic patients without neuropathic signs (17) and (b) there is excessive fat infiltration in leg skeletal muscles in individuals with obesity and DM (18). Since the muscle fiber bundles are composites of fibers and extracellular matrix (ECM) that bear the majority of passive muscle loads (19), the reduced shear-wave velocity or Young's modulus in muscles in this 
study may be related to reduced contractile and biochemical properties of muscle fibers (20); adverse remodeling of the ECM in diabetic muscle, including irregular organization and decreased matrix metalloproteinase (MMP) activation (21); and increases of intramuscular fat (18) in diabetes. The reduced muscle Young's modulus found in this study has three clinical implications: (a) insulin resistance and diabetes are detrimental to the mechanical properties of muscle fiber and ECM, muscle composition, and force production, an implication which is supported by the correlation between the calf muscle Young's modulus and the maximal heel lifting height in the present study; (b) the adaptive capacity of diabetic muscles for exercise or physical training may be reduced due to the diminished Young's modulus, which jeopardizes the force transmission, and the sensitivity of the muscle to mechanical stimulation (22); and (c) based on our findings showing that the medial gastrocnemius muscle has all of the aforementioned deterioration characteristics in diabetic muscles, the calf (distal) muscles of patients may develop diabetic myopathy earlier than the hip or thigh (proximal) muscles, and therefore, progressive strengthening and stretching exercises for leg muscles are strongly recommended for early detected or well-controlled diabetes. This exercise strategy is aimed at preventing muscle dysfunction and tendon pain. The benefits of exercises may include the promotion of ECM production and stiffness through mechanical stimulation (23). Although exercise might be beneficial to the turnover of ECM structure and could potentially lead to an attenuation of ECM accumulation as seen during chronic metabolic disease in humans (24), there have been few studies to illuminate differences in the effects of active and passive training on the muscle ECM. It is thus suggested that elastography be utilized in future studies to periodically measure the effects of exercise on the muscle ECM, to monitor deterioration in the muscle tissue, and to estimate the capacity of muscle force generation in diabetic patients.

The ultrasound results in this study regarding the microstructure (PSFR) of tendon tissue indicated that the diabetic tendons showed altered structural and composition characteristics in terms of packing density and alignment of the collagen bundles and fibrils consisting of changes of sizes, density, spatial alignments, and distribution (organization) (12). These results echo those of a previous study that reported increased packing density of collagen fibrils, decreases in fibrillar diameter, and abnormal fibril morphology consisting of twisted, curved, overlapping, and otherwise highly disorganized fibrils observed by electron microscopy in the Achilles tendons of diabetic patients (4). Combined with the results regarding correlations between the PSFR and the $\mathrm{HbA1c}$ or HDL-C levels found in this study, these morpho- logic abnormalities of the collagen bundles and fibrils in diabetic tendons appear to be the result of nonenzymatic glycation and the production of inflammatory cytokines expressed over the years in those suffering from DM $(4,25)$. These microstructural changes are suggested to be the causes of reduced tendon flexibility, tendon thickening, and shortening of the Achilles tendon-gastrocnemius complex, as well as tendinopathy $(2,4)$. The ultrasound characteristics of the quadriceps, patellar, and Achilles tendons found in this study indicated the early detection of tendon microstructural changes in diabetes, and suggest that microstructural abnormalities may begin prior to the occurrence of symptoms or macrostructural changes in diabetic tendons, such as tendon thickening and reduced tendon stiffness (2). Potential applications of the technology used in the current study thus include screening for the risk of tendinopathy in diabetic patients while they are still asymptomatic. The results of this study support the conclusions that diabetic muscles and tendons exhibit early mechanical or structural changes, including reduced muscle Young's modulus and PSFR in the tendons, and that the mechanical or structural changes in the Achilles tendon-gastrocnemius complex are associated with force capacity or markers of insulin resistance.

\section{LIMITATIONS}

This study limitations include the specific diabetic population studied, such that the results must be interpreted with caution with respect to using well-controlled diabetes as a reference for the tendon system. In addition, this study did not analyze the effects of commonly prescribed drugs (including statins) that may affect the collagen structure and PSFR in patients with DM. Moreover, our results may not apply to diabetes with poor control or peripheral neurovascular disease.

\section{Clinical implication}

Based on our study results, which were gathered from participants who had no peripheral neurovasculopathy, strengthening and stretching exercises for the calf muscles are strongly recommended for prophylactic programs aimed at the prevention of lower extremity dysfunction. It is suggested that elastography and the scattering pattern approach be used in future studies to reveal the training effects of calf exercises and to verify the central role of mechanical and morphological plasticity in functional recovery in diabetic participants.

\section{CONFLICT OF INTERESTS}

The authors declare that they have no conflict of interests. 


\section{REFERENCES}

1. Merashli M, Chowdhury TA, Jawad AS. Musculoskeletal manifestations of diabetes mellitus. QJM 2015;108:853-857.

2. Oliva F, Piccirilli E, Berardi AC, Frizziero A, Tarantino $\mathrm{U}$, Maffulli N. Hormones and tendinopathies: the current evidence. Br Med Bull 2016;117:39-58.

3. Karampinos DC, Baum T, Nardo L, Alizai H, Yu H, Carballido-Gamio J, Yap SP, Shimakawa A, Link TM, Majumdar S. Characterization of the regional distribution of skeletal muscle adipose tissue in type 2 diabetes using chemical shift-based water/fat separation. J Magn Reson Imaging 2012;35:899-907.

4. Grant WP, Sullivan R, Sonenshine DE, Adam M, Slusser JH, Carson KA, Vinik AI. Electron microscopic investigation of the effects of diabetes mellitus on the Achilles tendon. J Foot Ankle Surg 1997;36:272-278.

5. Soliman SB, Rosen KA, Williams PC, Spicer PJ, Williams LK, Rao SD, van Holsbeeck MT. The hyperechoic appearance of the deltoid muscle on shoulder ultrasound imaging as a predictor of diabetes and prediabetes. J Ultrasound Med 2020;39:323-329.

6. Ursini F, Arturi F, D’Angelo S, Amara L, Nicolosi K, Russo E, Naty S, Bruno C, De Sarro G, Olivieri I, Grembiale RD. High prevalence of Achilles tendon enthesopathic changes in patients with type 2 diabetes without peripheral neuropathy. J Am Podiatr Med Assoc 2017;107:99-105.

7. Coombes BK, Tucker K, Hug F, Scott A, Geytenbeek M, Cox ER, Gajanand T, Coombes JS. Relationships between cardiovascular disease risk factors and Achilles tendon structural and mechanical properties in people with Type 2 Diabetes. Muscles Ligaments Tendons J 2019;9:395-404.

8. Guney A, Vatansever F, Karaman I, Kafadar IH, Oner M, Turk CY. Biomechanical properties of Achilles tendon in diabetic vs. non-diabetic patients. Exp Clin Endocrinol Diabetes 2015;123:428-432.

9. Kannus P. Structure of the tendon connective tissue. Scand J Med Sci Sports 2000;10:312-320.

10. Eby SF, Song P, Chen S, Chen Q, Greenleaf JF, An KN. Validation of shear wave elastography in skeletal muscle. J Biomech 2013;46:2381-2387.

11. Stetson P, Sommer G. Ultrasonic characterization of tissues via backscatter frequency dependence. Ultrasound Med Biol 1997;23:989-996.

12. Bashford GR, Tomsen N, Arya S, Burnfield JM, Kulig K. Tendinopathy discrimination by use of spatial frequency parameters in ultrasound B-mode images. IEEE Trans Med Imaging 2008;27:608-615.
13. Davis PJ, Liu M, Sherman S, Natarajan S, Alemi F, Jensen A, Avramovic S, Schwartz MD, Hayes RB. HbA1c, lipid profiles and risk of incident type 2 Diabetes in United States Veterans. PLoS One 2018;13:e0203484.

14. Padulo J., Oliva F., Frizziero A., Maffulli N. Muscles, Ligaments and Tendons Journal - Basic principles and recommendations in clinical and field Science Research: 2018 update. Muscles Ligaments Tendons J 2018; 8:305-307.

15. American Diabetes Association. 2. Classification and Diagnosis of Diabetes: Standards of Medical Care in Diabetes-2018. Diabetes Care 2018;41:S13-S27.

16. Haffner SM; American Diabetes Association. Dyslipidemia management in adults with diabetes. Diabetes Care 2004:27:S68-71.

17. Severinsen K, Obel A, Jakobsen J, Andersen H. Atrophy of foot muscles in diabetic patients can be detected with ultrasonography. Diabetes Care 2007;30:3053-3057.

18. Hilton TN, Tuttle LJ, Bohnert KL, Mueller MJ, Sinacore DR. Excessive adipose tissue infiltration in skeletal muscle in individuals with obesity, diabetes mellitus, and peripheral neuropathy: association with performance and function. Phys Ther. 2008;88:1336-1344.

19. Gillies AR, Lieber RL. Structure and function of the skeletal muscle extracellular matrix. Muscle Nerve 2011;44:318-331.

20. Stephenson GM, O'Callaghan A, Stephenson DG. Single-fiber study of contractile and biochemical properties of skeletal muscles in streptozotocin-induced diabetic rats. Diabetes 1994:43:622-628.

21. Ahmad K, Lee EJ, Moon JS, Park SY, Choi I. Multifaceted Interweaving Between Extracellular Matrix, Insulin Resistance, and Skeletal Muscle. Cells 2018;7: pii: E148.

22. Wisdom KM, Delp SL, Kuhl E. Use it or lose it: multiscale skeletal muscle adaptation to mechanical stimuli. Biomech Model Mechanobiol 2015;14:195-215.

23. Bezerra MA, da Silva Nery C, de Castro Silveira PV, et al. Previous physical exercise slows down the complications from experimental diabetes in the calcaneal tendon. Muscles Ligaments Tendons J 2016;19;6(1):97-103.

24. Martinez-Huenchullan S, McLennan SV, Verhoeven A, Twigg SM, Tam CS. The emerging role of skeletal muscle extracellular matrix remodeling in obesity and exercise. Obes Rev 2017;18:776-790.

25. Yang Y, Lu H, Qu J. Tendon pathology in hypercholesterolaemia patients: Epidemiology, pathogenesis and management. J Orthop Translat 2018;16:14-22. 